\title{
Option Pricing in Subdiffusive Bachelier Model
}

\author{
Marcin Magdziarz • Sebastian Orzel • \\ Aleksander Weron
}

Received: 21 April 2011 / Accepted: 9 August 2011 / Published online: 9 September 2011

(C) The Author(s) 2011. This article is published with open access at Springerlink.com

\begin{abstract}
The earliest model of stock prices based on Brownian diffusion is the Bachelier model. In this paper we propose an extension of the Bachelier model, which reflects the subdiffusive nature of the underlying asset dynamics. The subdiffusive property is manifested by the random (infinitely divisible) periods of time, during which the asset price does not change. We introduce a subdiffusive arithmetic Brownian motion as a model of stock prices with such characteristics. The structure of this process agrees with two-stage scenario underlying the anomalous diffusion mechanism, in which trapping random events are superimposed on the Langevin dynamics. We find the corresponding fractional Fokker-Planck equation governing the probability density function of the introduced process. We construct the corresponding martingale measure and show that the model is incomplete. We derive the formulas for European put and call option prices. We describe explicit algorithms and present some Monte-Carlo simulations for the particular cases of $\alpha$-stable and tempered $\alpha$-stable distributions of waiting times.
\end{abstract}

Keywords Subdiffusion · Fractional Fokker-Planck equation · Bachelier model · Option pricing · Model of financial markets · Infinitely divisible distribution · Tempered stable distribution

\section{Introduction}

The modern approach to financial engineering based on continuous-time processes began in the year 1900 with the pioneering thesis of Louis Bachelier, [1], "Théorie de la Spéculation". Probably the most remarkable achievement of Bachelier was the development of the

M. Magdziarz · S. Orzeł $(\bowtie) \cdot$ A. Weron

Hugo Steinhaus Center, Institute of Mathematics and Computer Science, Wrocław University of Technology, Wyb. Wyspiańskiego 27, 50-370 Wrocław, Poland e-mail: Sebastian.Orzel@pwr.wroc.pl

M. Magdziarz

e-mail: Marcin.Magdziarz@pwr.wroc.pl

A. Weron

e-mail: Aleksander.Weron@pwr.wroc.pl 
mathematical theory of Brownian motion. He initiated the study of diffusion processes five years before the famous physical papers by Albert Einstein, [14] and Marian Smoluchowski, [38], and decades before the works of mathematicians Norbert Wiener, Kiyoshi Itô and Paul Lévy [16, 21, 42]. Bachelier observed that the stock price movements are analogous to the motion of small particles suspended in liquids. With this assumption he derived the underlying equations of motion and found the pricing formulas for put and call options on such stocks. Let us add here that, in accordance with tradition at Sorbonne, he also defended a second thesis on mechanics of fluids [8].

In the contemporary terms, Bachelier assumed that the stock price follows an arithmetic Brownian motion (ABM). Although the trajectories of ABM can become negative, the Bachelier model appears to be an appropriate tool to handle short-lived derivatives [28]. Moreover, it has been demonstrated recently [37], that option pricing formulas of Bachelier and Black-Scholes coincide very well in the sense that Bachelier's model yields good short time approximations of prices and volatilities.

In dissertation [1] and subsequent works Bachelier anticipated much of what was to become standard fare in stochastic calculus, diffusion theory and mathematical finance: random walk of market price, limit of random walks, Brownian motion, and martingale measure. The innovative ideas of Bachelier were much above the prevailing level of existing financial theory, physics and mathematics. For example, he calculated the probability that the Brownian motion does not exceed a fixed level and found the distribution of the supremum of the Brownian motion. Even more surprisingly, he derived the distribution of the Ornstein-Uhlenbeck process In spite of its mathematical elegance and novelty, the theory of Bachelier was neglected for over fifty years. Samuelson, [36], was the first to rediscover Bachelier's ideas to introduce a more adequate description of stock price dynamics based on the geometric Brownian motion (GBM). The culminating point in the extensive research on GBM in the context of financial engineering, was the result of Black, Scholes and Merton $[4,26]$, who derived consistent formulas for the fair prices of European options. Their discovery was of such great importance that Merton and Scholes were awarded the Nobel Prize for Economics in 1997. After the recent findings [37], it seems that the Black-ScholesMerton success was overestimated. From the physical point of view the Bachelier approach is more natural. Since the probability density function (PDF) corresponding to ABM satisfies the well-known diffusion-advection equation [27].

Analysis of miscellaneous financial markets shows that majority of economical processes are far away from the classical diffusion models considered in financial mathematics. Hence, in recent years we observe an increasing number of mathematical models which deal with non-ideal properties of real-life financial markets. For example, Black-Scholes model and other Lévy type models [7] are incapable of adequately fitting implied volatility surfaces of equity options across both strike and maturities. In order to overcome this difficulty during the last decade dozens of alternative models have been proposed within the class of H-selfsimilar models, see [15].

The often encountered property of financial data are the characteristic periods of time in which economic process stays motionless (periods of stagnation). Such behavior is typical for emerging markets with low number of transactions [17] and for interest rate markets [30]. For interest-rate, currency, and commodity markets, see [18]. Notably, analogous behavior is observed in complex physical systems, in which the motion of small particles is interrupted by the trapping events - the random periods of time when the particle gets immobilized in a trap. Real-life examples of subdiffusive dynamics include: charge carrier transport in amorphous semiconductors, nuclear magnetic resonance, diffusion in percolative and porous systems, transport on fractal geometries and dynamics of a bead in a polymeric network, as well as protein conformational dynamics (see [27] and references therein). 
Following the same line as Bachelier, we infer that the constant periods of stagnation in financial processes are analogous in nature to the trapping events of the subdiffusive particle. Therefore, the physical models of subdiffusion can be successfully applied to describe financial data.

The usual model of subdiffusion in physics is the celebrated Fractional Fokker-Planck equation (FFPE). This equation was derived from the continuous-time random walk scheme with heavy-tailed waiting times (see [27]). Since then it became the standard mathematical tool in the analysis of complex systems, [10-13]. Equivalent description of subdiffusion is in terms of subordination, where the standard diffusion process is time-changed by the so-called inverse subordinator $[22,25]$. Following this line, in this paper we introduce a subdiffusive ABM as a model of stock prices. This process is defined as the standard ABM subordinated by the infinitely divisible (id) inverse subordinator. For a historical review on financial models exploiting the method of subordination, see [15]. Since the id inverse subordinators do not have independent increments, the examined here process does not belong to the extensively studied class of subordinated additive processes $[6,15]$, which has found widespread applications in finance.

We underline that the presented here methodology can be easily extended to other diffusion processes time-changed by the inverse subordinators. For the sake of clarity and simplicity, we consider only ABM. Analogous results can be obtained for other diffusion processes (see $[23,39]$ for the case of GBM with $\alpha$-stable waiting times).

The paper is organized as follows. In the next section we give the precise definition of subdiffusive ABM, and find the corresponding fractional Fokker-Planck equation governing the dynamics of PDF. In Sect. 3 we prove that the model is arbitrage-free and incomplete. We also derive formulas for the fair prices of European options in our model. Finally, we consider two particular examples of waiting-time distributions: $\alpha$-stable and tempered $\alpha$-stable. We discuss the methods of simulation of the trajectories of subdiffusive ABM and present some numerical results illustrating our findings.

\section{Subdiffusive Model of Stock Prices}

In modern terms, in the Bachelier model the stock price dynamics follows $\mathrm{ABM}$

$$
X(t)=X_{0}+\mu t+\sigma B(t), \quad t \geq 0 .
$$

Here, $\mu \in \mathbb{R}$ is the drift parameter, $\sigma \in \mathbb{R}_{+}$is the volatility and $B(t)$ is the standard Brownian motion (Wiener process) on the probability space $(\Omega, \mathcal{F}, \mathbb{P})$. This market model is arbitragefree and complete.

Option pricing formulas in the Bachelier model can be derived using the standard martingale method. Considering a European call option on a stock $X$ with expiry date $T$ and strike price $K$, one can show that its fair price is given by [28]

$$
C\left(X_{0}, K, T, \sigma\right)=\sigma \sqrt{T} n\left(\frac{X_{0}-K}{\sigma \sqrt{T}}\right)+\left(X_{0}-K\right) N\left(\frac{X_{0}-K}{\sigma \sqrt{T}}\right) .
$$

Here, $n$ (respectively $N$ ) is the PDF (respectively, cumulative distribution function) of the standard normal distribution. We assume for simplicity that the risk-free interest rate $r=0$, which is common for the Bachelier model (see [28]). By the put-call parity, the price of the put option yields

$$
P\left(X_{0}, K, T, \sigma\right)=C\left(X_{0}, K, T, \sigma\right)+K-X_{0}
$$


The following inequality between Bachelier price $C^{B}=C\left(X_{0}, K, T, \sigma\right)$ and the BlackScholes price $C^{B S}$ was obtained in [37]

$$
0 \leq C^{B}-C^{B S} \leq \frac{X_{0}}{12 \sqrt{2}} \sigma^{B} T^{3 / 2}
$$

where $\sigma^{B}$ is the implied volatility in the Bachelier model. Moreover, the difference between the implied volatilities in both models was estimated in [37] as follows

$$
0 \leq \sigma^{B S}-\sigma^{B} \leq \frac{T}{12}\left(\sigma^{B S}\right)^{3} .
$$

This demonstrates how close the Bachelier and the Black-Scholes option pricing formulas are. Now, our goal is to extend the Bachelier model in order to capture the subdiffusive character of the underlying asset (the random periods of stagnation in which the price does not change). Recall that this property is particularly visible in the emerging markets, in which the number of transactions is low. We begin with defining the so-called inverse subordinator. It is defined as

$$
S_{\Psi}(t)=\inf \left\{\tau>0: T_{\Psi}(\tau)>t\right\} .
$$

Here, $T_{\Psi}(t)$ is the strictly increasing Lévy process [3] with the Laplace transform

$$
\left\langle\exp \left(-u T_{\Psi}(t)\right)\right\rangle=\exp (-t \Psi(u)) .
$$

The Laplace exponent is given by $\Psi(t)=\int_{0}^{\infty}\left(1-e^{-u x}\right) v(d x)$ with the Lévy measure $v$ satisfying $\int_{0}^{\infty}(1 \wedge x) v(d x)<\infty$. To exclude the case of compound Poisson process, we additionally assume that $v(0, \infty)=\infty$. Contrary to $T_{\Psi}(t)$ which is a pure jump process, the trajectories of $S_{\Psi}(t)$ are continuous. Moreover, they are singular with respect to the Lebesgue measure. Additionally, for every jump of $T_{\Psi}(t)$ there is a corresponding flat period of its inverse. These flat periods, distributed according to id law, are characteristic for the subdiffusive dynamics and they represent the waiting times in which the test particle gets immobilized in the trap.

Now, in analogy with the physical description of subdiffusion, we introduce the subdiffusive $\mathrm{ABM}$

$$
Y_{\Psi}(t)=X\left(S_{\Psi}(t)\right)=X_{0}+\mu S_{\Psi}(t)+\sigma B\left(S_{\Psi}(t)\right),
$$

$t \in[0, T]$, as a model of stock prices. Here, the processes $X(t)$ and $S_{\Psi}(t)$ are assumed independent. The typical trajectory of $Y_{\Psi}(t)$ with tempered $\alpha$-stable waiting times (i.e. $\left.\Psi(u)=(u+\lambda)^{\alpha}-\lambda^{\alpha}, 0<\alpha<1, \lambda>0\right)$ is shown in Fig. 1. The main difference between the classical and subdiffusive ABM are the constant periods in the realizations of the latter process. Depending on the choice of the exponent $\Psi(u)$, we obtain different id distributions of the waiting times in which the price does not change. This assures flexibility and universality of the introduced model. Important examples of id laws include: $\alpha$-stable, Pareto, exponential, gamma, Linnik, Mittag-Leffler, and tempered $\alpha$-stable distributions, [7].

Now, we present the fractional Fokker-Planck equation governing the PDF of $Y_{\Psi}(t)$. This type of equation is the usual tool used in physics to analyze subdiffusive dynamics. PDF of the process $Y_{\Psi}(t)$ is the solution of the following fractional Fokker-Planck equation

$$
\frac{\partial w(x, t)}{\partial t}=\Phi_{t}\left[-\mu \frac{\partial}{\partial x}+\frac{\sigma^{2}}{2} \frac{\partial^{2}}{\partial x^{2}}\right] w(x, t),
$$


Fig. 1 Sample realizations of the standard ABM $X(t)$ (top panel), and the corresponding subdiffusive ABM $Y_{\Psi}(t)=X\left(S_{\Psi}(t)\right)$ with tempered stable waiting times (bottom panel). The constant intervals of the latter process are typical for subdiffusion and represent the periods of stagnation
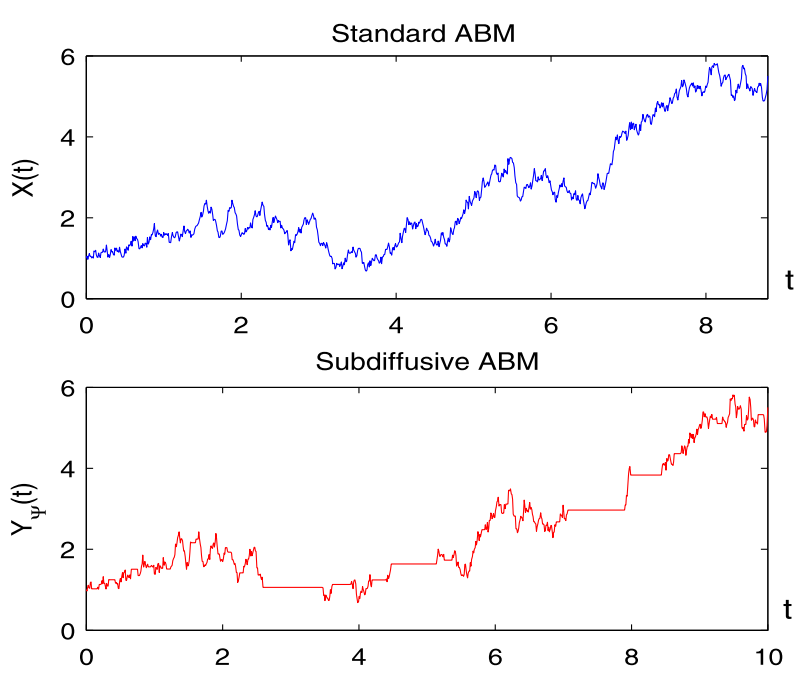

$w(x, 0)=\delta\left(x-X_{0}\right)$. Here $\Phi_{t}$ is the integro-differential operator defined as

$$
\Phi_{t} f(t)=\frac{d}{d t} \int_{0}^{t} M(t-y) f(y) d y,
$$

with the memory kernel $M(t)$ defined via its Laplace transform

$$
\widehat{M}(u)=\int_{0}^{\infty} e^{-u t} M(t) d t=\frac{1}{\Psi(u)} .
$$

For the derivation of formula (7), see Appendix A. The above result allows to find, at least in some particular cases, closed-form formulas for the PDF of $Y_{\Psi}(t)$. Moreover, approximated solutions of (7) can be derived by the finite element method (see [9]), or by the Monte Carlo techniques based on the simulation algorithm of $Y_{\Psi}(t)$ (see Sect. 4).

\section{Lack of Arbitrage, Incompleteness and Option Pricing Formula}

Let us consider a market, whose evolution up to time horizon $T$ is contained in the probability space $(\Omega, \mathcal{F}, \mathbb{P})$. The asset price in this market is described by the subdiffusive ABM $Y_{\Psi}(t)$. Denote by $\left(\mathcal{F}_{t}\right)_{t \in[0, T]}$ the filtration generated by $Y_{\Psi}(t)$, i.e. history of the process up to time $t$. The usual requirement for pricing rules in a given market model is that it does not admit arbitrage opportunities. Recall that to assure that the market model is arbitrage-free, it is enough to prove the existence of the equivalent martingale measure, see [7]. The martingale property formalize the concept of the fairness of the game, i.e. its increment process is a fair-game, meaning that the expectation value of increments is zero, for more details and connection to anomalous diffusion see [41].

Let us introduce the following measure

$$
\mathbb{Q}(A)=\int_{A} \exp \left\{-\gamma B\left(S_{\Psi}(T)\right)-\frac{\gamma^{2}}{2} S_{\Psi}(T)\right\} d \mathbb{P},
$$


where $\gamma=\frac{\mu}{\sigma}$ and $A \in \mathcal{F}$. Clearly, $\mathbb{Q}$ is a probability measure equivalent to $\mathbb{P}$. Moreover, in Appendix B it is proved that the subdiffusive $\mathrm{ABM} Y_{\Psi}(t)$ is a martingale with respect to $\mathbb{Q}$. Consequently, by the Fundamental theorem of asset pricing [7] we obtain that the market model, in which the asset price is described by the subdiffusive ABM, is arbitrage-free.

Another typical question raised in the context of market model is the question of its completeness. Recall that the market is complete if every $\mathcal{F}_{T}$-measurable contingent claim admits a replicating self-financing strategy [7]. Completeness of a market is usually verified in the framework of the martingale methodology. The Second Fundamental theorem of asset pricing states that the model is complete if and only if there is a unique martingale measure. In Appendix $\mathrm{C}$ we give two very different proofs of the incompleteness of financial market model based on subdiffusive $\mathrm{ABM} Y_{\Psi}(t)$. In the first one the whole family of equivalent martingale measures is constructed, whereas in the second proof we show that not every contingent claim can be replicated. Both proofs are of independent interest, therefore we decided to insert each of them.

The next step is the derivation of the option pricing formulas. Here, we will concentrate on the European options, however, other derivatives can be priced in a similar manner. From Appendix $\mathrm{C}$ we know that the subdiffusive model is incomplete. As a consequence, we obtain different prices of derivatives depending on the choice of the martingale measure. In what follows, we will concentrate only on the measure $\mathbb{Q}$ defined in (8), since it is the natural extension of the martingale measure from the classical Bachelier model, in the context of subordination. Moreover, the relative entropy (also called the Kullback-Leibler divergence [20]) of $\mathbb{Q}$ is smaller than the relative entropy of measures $\mathbb{Q}_{\epsilon}$ defined in (20). This means that the distance between measure $\mathbb{Q}$ and the "real-life" measure $\mathbb{P}$ is smallest in terms of relative entropy. The relative entropy of $\mathbb{Q}$ is equal to

$$
D=-\int_{\Omega} \log \frac{d \mathbb{Q}}{d \mathbb{P}} d \mathbb{P}=\frac{\gamma^{2}}{2}\left\langle S_{\Psi}(T)\right\rangle .
$$

On the other hand, the relative entropy of $\mathbb{Q}_{\epsilon}$ yields

$$
\begin{aligned}
D_{\epsilon}= & -\int_{\Omega} \log \frac{d \mathbb{Q}_{\epsilon}}{d \mathbb{P}} d \mathbb{P}=\log \left\langle\exp \left\{-\gamma B\left(S_{\Psi}(T)\right)-\left(\epsilon+\frac{\gamma^{2}}{2}\right) S_{\Psi}(T)\right\}\right\rangle \\
& +\left(\epsilon+\frac{\gamma^{2}}{2}\right)\left\langle S_{\Psi}(T)\right\rangle=\log \left\langle\exp \left\{-\epsilon S_{\Psi}(T)\right\}\right\rangle+\left(\epsilon+\frac{\gamma^{2}}{2}\right)\left\langle S_{\Psi}(T)\right\rangle \\
\geq & \frac{\gamma^{2}}{2}\left\langle S_{\Psi}(T)\right\rangle=D .
\end{aligned}
$$

This confirms that the measure $\mathbb{Q}$ minimizes the relative entropy.

It is important to emphasize here that although the recipes for obtaining option prices are the same for Bachelier's as for the Black-Scholes approach, the arguments in favor of them are very different. Namely, an equilibrium argument in Bachelier case, as opposed to the no arbitrage arguments in the Black-Scholes approach, [37].

Consider the market model, in which the asset price follows the subdiffusive $\mathrm{ABM} Y_{\Psi}(t)$. Further, let the martingale measure $\mathbb{Q}$ be defined in (8). Then, the corresponding fair price of the European call option with expiry date $T$ and strike price $K$ is given by

$$
\begin{aligned}
C_{\Psi}\left(X_{0}, K, T, \sigma\right) & =\left\langle C\left(X_{0}, K, S_{\Psi}(T), \sigma\right)\right\rangle \\
& =\int_{0}^{\infty} C\left(X_{0}, K, x, \sigma\right) g_{\Psi}(x, T) d x .
\end{aligned}
$$


Here, $g_{\Psi}(x, T)$ is the PDF of $S_{\Psi}(T)$ and $C\left(X_{0}, K, T, \sigma\right)$ is given by (2).

The above formula follows from the fact that

$$
\begin{aligned}
C_{\Psi}\left(X_{0}, K, T, \sigma\right) & =\left\langle\left(Y_{\Psi}(T)-K\right)^{+}\right\rangle_{\mathbb{Q}} \\
& =\left\langle\exp \left\{-\gamma B\left(S_{\Psi}(T)\right)-\frac{\gamma^{2}}{2} S_{\Psi}(T)\right\}\left(Y_{\Psi}(T)-K\right)^{+}\right\rangle,
\end{aligned}
$$

where $\langle.\rangle_{\mathbb{Q}}$ denotes expectation with respect to the martingale measure $\mathbb{Q}$. Therefore, conditioning on $S_{\Psi}(T)$, we obtain

$$
C_{\Psi}\left(X_{0}, K, T, \sigma\right)=\left\langle C\left(X_{0}, K, S_{\Psi}(T), \sigma\right)\right\rangle=\int_{0}^{\infty} C\left(X_{0}, K, x, \sigma\right) g_{\Psi}(x, T) d x,
$$

where $g_{\Psi}(x, T)$ is the PDF of $S_{\Psi}(T)$. This ends the proof of formula (9).

The price of the corresponding European put option is easily recovered from the put-call parity (3)

$$
P_{\Psi}\left(X_{0}, K, T, \sigma\right)=C_{\Psi}\left(X_{0}, K, T, \sigma\right)+K-X_{0}
$$

There are two ways of finding the values of the price $C_{\Psi}(\cdot)$. The first one is by the Monte Carlo method. One simulates trajectories of the inverse subordinator $S_{\Psi}(t)$ on the interval $[0, T]$ and calculates the expected value in (9). The following efficient approximation scheme [22] can be used to simulate numerically the trajectories of $S_{\Psi}(t)$

$$
S_{\Psi, \delta}(t)=\left(\min \left\{n \in \mathbb{N}: T_{\Psi}(\delta n)>t\right\}-1\right) \delta .
$$

Here, $\delta>0$ is the step length and $T_{\Psi}(\tau)$ is the subordinator introduced in (5). As shown in [22], $S_{\Psi, \delta}(\cdot)$ converges uniformly and strongly to $S_{\Psi}(\cdot)$ as $\delta \searrow 0$. To simulate $S_{\Psi, \delta}(t)$, one only needs to generate the values $T_{\Psi}(\delta n), n=1,2, \ldots$ Since $T_{\Psi}(t)$ is a Lévy process, this can be done by the general method presented in [33]. For some particular cases of id distributions, more efficient algorithms are at hand (see Sect. 4).

The second way of finding $C_{\Psi}(\cdot)$ is by approximating the integral in (9). However, this can be performed only in the cases, when the PDF $g_{\Psi}(x, T)$ is known explicitly. Some particular examples are presented in Sect. 4.

\section{Examples of Waiting Times}

\section{$4.1 \alpha$-Stable Waiting Times}

The first considered here example of waiting-time distribution is the $\alpha$-stable law, which is the most prominent representative of the class of heavy-tailed distributions ([19,35]). In the $\alpha$-stable case the Laplace exponent in (5) is given by

$$
\Psi(u)=u^{\alpha}, \quad 0<\alpha<1 .
$$

Consequently, $T_{\Psi}(t)$ is the $1 / \alpha$-self-similar stable subordinator. Therefore, the subordinated Brownian motion $B\left(S_{\Psi}(t)\right)$ is $\alpha / 2$-self-similar. This desired feature plays an important role in the modeling of financial markets ([15]), it also simplifies significantly all the calculations in the $\alpha$-stable case. 


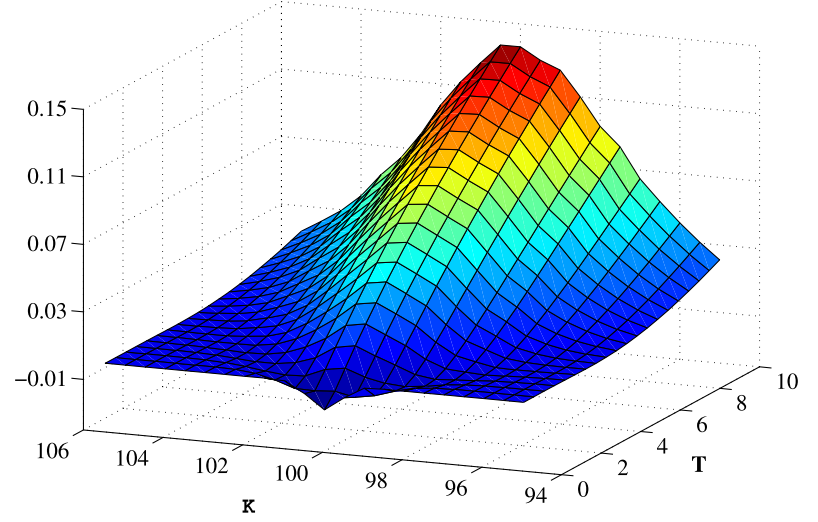

Fig. 2 The difference between the classical Bachelier price of the European call option and the subdiffusive one $C\left(X_{0}, K, T, \sigma\right)-C_{\Psi}\left(X_{0}, K, T, \sigma\right)$, according to the exercise date $T$ and strike price $K$. In the subdiffusive model the waiting times follow the $\alpha$-stable law $\left(\Psi(u)=u^{\alpha}\right)$. Here, $X_{0}, \sigma$ and $\alpha$ are fixed and equal 100,1 and 0.9 , respectively. For small $T$ the subdiffusive price is larger than the classical Bachelier price (the difference is negative), while for the larger $T$ the subdiffusive price is smaller than the Bachelier one

Additionally, the operator $\Phi_{t}$ in formula (7) reduces to the fractional derivative of the Riemann-Liouville type [34]

$$
{ }_{0} D_{t}^{1-\alpha} f(t)=\frac{1}{\Gamma(\alpha)} \frac{d}{d t} \int_{0}^{t}(t-s)^{\alpha-1} f(s) d s,
$$

and the solution of (7) can be represented in terms of the special Fox function (see [27]). Moreover, the PDF $g_{\Psi}(x, T)$ of the inverse $\alpha$-stable subordinator $S_{\Psi}(t)$ yields

$$
g_{\Psi}(x, T)=T^{-\alpha} g_{\alpha}\left(x / T^{\alpha}\right),
$$

where $g_{\alpha}(z)=H_{11}^{10}\left(\left.z\right|_{(0,1)} ^{(1-\alpha, \alpha)}\right)$ is the Fox function [34]. In particular, for $\alpha=0.5$ we get that $g_{0.5}(z)=\frac{1}{\sqrt{\pi}} \exp \left(-\frac{z^{2}}{4}\right)$, which allows to evaluate numerically integral (9) and to find the fair prices of call and put options.

In the $\alpha$-stable case, the approximation scheme (11) works very efficiently. The values $T_{\Psi}(\delta n), n=1,2, \ldots$, in (11) can be generated by the standard Euler method [19]:

$$
\begin{aligned}
& T_{\Psi}(0)=0, \\
& T_{\Psi}(\delta n)=T_{\Psi}(\delta(n-1))+\delta^{1 / \alpha} \xi_{n},
\end{aligned}
$$

where $\xi_{n}, n \in \mathbb{N}$, are the i.i.d. positive $\alpha$-stable random variables. The method of generating $\xi_{n}$ is the following [40]

$$
\xi_{n}=\frac{\sin \left(\alpha\left(V+c_{1}\right)\right)}{(\cos (V))^{1 / \alpha}}\left(\frac{\cos \left(V-\alpha\left(V+c_{1}\right)\right)}{W}\right)^{(1-\alpha) / \alpha},
$$

where $c_{1}=\pi / 2, V$ is the uniformly distributed on $(-\pi / 2, \pi / 2)$ random variable and $W$ has exponential distribution with mean one.

The above algorithm allows to simulate efficiently trajectories of $S_{\Psi}(t)$. Therefore, Monte Carlo methods can be employed to approximate the price $C_{\Psi}(\cdot)$ in (9). In Fig. 2 
we present the results of the Monte Carlo simulations. Shown is the difference between the classical Bachelier price of the European call option and the subdiffusive one $C\left(X_{0}, K, T, \sigma\right)-C_{\Psi}\left(X_{0}, K, T, \sigma\right)$.

\subsection{Tempered $\alpha$-Stable Waiting Times}

The class of tempered stable distributions was introduced in [32]. It leads to an appropriate modification of the Lévy measure corresponding to $\alpha$-stable law, which resulted in many desired properties of tempered laws. In particular, tempered $\alpha$-stable distributions are infinitely divisible. Moreover, they have finite moments of all orders, which makes them particularly attractive for applications. On the other hand, tempered stable distributions resemble stable laws in many aspects (see [32] for the details).

For the case of tempered $\alpha$-stable waiting times, the Laplace exponent in (5) is given by

$$
\Psi(u)=(u+\lambda)^{\alpha}-\lambda^{\alpha}, \quad 0<\alpha<1, \lambda>0 .
$$

Note that for $\lambda \searrow 0$, we recover the Laplace exponent of $\alpha$-stable distribution.

As for the operator $\Phi_{t}$ in (7), its inverse superposed with the standard derivative $\Phi_{t}^{-1} \circ \frac{\partial}{\partial t}$ is the tempered fractional derivative, which was originally developed in the paper [5]. This allows to apply the finite element method (see [9]) to approximate solutions of (7).

As for the algorithm of simulation of $S_{\Psi}(t)$, it is very similar to the $\alpha$-stable case. The values $T_{\Psi}(\delta n), n=1,2, \ldots$, in (11) are generated by the standard Euler method

$$
\begin{aligned}
& T_{\Psi}(0)=0, \\
& T_{\Psi}(\delta n)=T_{\Psi}(\delta(n-1))+Z_{n},
\end{aligned}
$$

where $Z_{n}$ are independent, identically distributed tempered $\alpha$-stable random variables with the Laplace transform

$$
\left\langle e^{-u Z_{n}}\right\rangle=e^{-\delta\left((u+\lambda)^{\alpha}-\lambda^{\alpha}\right)} .
$$

The algorithm of generating $Z_{n}$ is the following [2]:

(I) Generate exponential random variable $E$ with mean $\lambda^{-1}$;

(II) Generate positive $\alpha$-stable random variable $S$ using formula (12) and put $R=\delta^{1 / \alpha} S$;

(III) If $E>R$ put $Z_{n}=R$, otherwise goto step (I).

The results of the above algorithm are presented in Fig. 1. We observe a typical trajectory of ABM with tempered stable waiting times. Similarly to the stable case, one can use the above algorithm to perform Monte Carlo simulations in order to approximate the price $C_{\Psi}(\cdot)$ in (9), see Figs. 2-6.

\section{Concluding Remarks}

In this paper we have applied the recent advances in the theory of anomalous diffusion to the problem of option pricing. We have introduced an extension of the classical Bachelier model, which captures the subdiffusive character of the underlying asset. The model is defined as the standard ABM subordinated by the id inverse subordinator. This assures the flexibility of the model, since the periods of stagnation of the price process can be chosen from the 


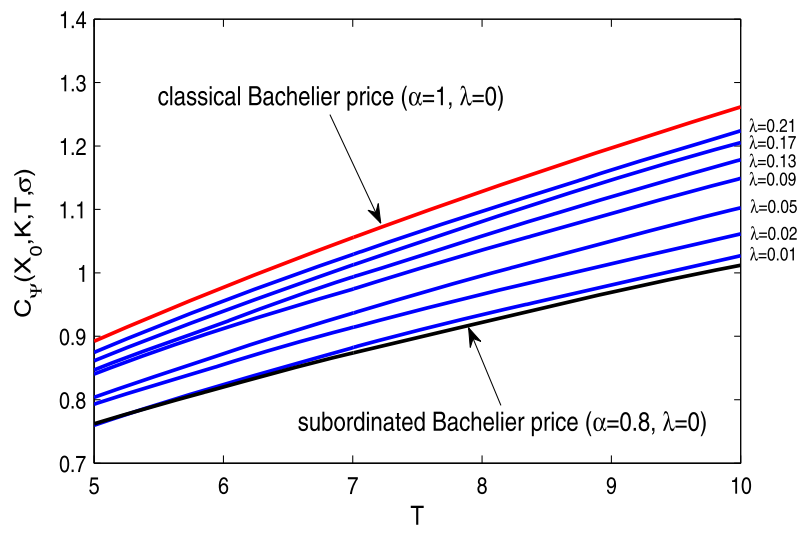

Fig. 3 (Color online) The comparison of the Bachelier prices of the European call option $C_{\Psi}\left(X_{0}, K, T, \sigma\right)$, according to the $\alpha$ and $\lambda$ parameters. Tempered stable distribution take intermediate place between pure stable and Gaussian distributions, as one can see, such behavior is common also for option prices. For $\lambda \searrow 0$ option prices with tempered subordinator (blue lines, $\alpha=0.8, \lambda>0$ ) tend to the option price with pure stable subordinator (black line, $\alpha=0.8, \lambda=0$ ). In turn, for large $\lambda$ they tend to the classical Bachelier price (red line, $\alpha=1, \lambda=0)$. Here, $X_{0}, K, \sigma$ are fixed and equal $100,100,1$, respectively and $T \in[5,10]$

Fig. 4 Bachelier prices of European call option $C_{\Psi}\left(X_{0}, K, T, \sigma\right)$, according to the $\alpha$ parameter. Here, $\lambda, X_{0}, K$, $\sigma$ are fixed and equal $0.001,100$, 100,1 , respectively and $T \in[0,10]$

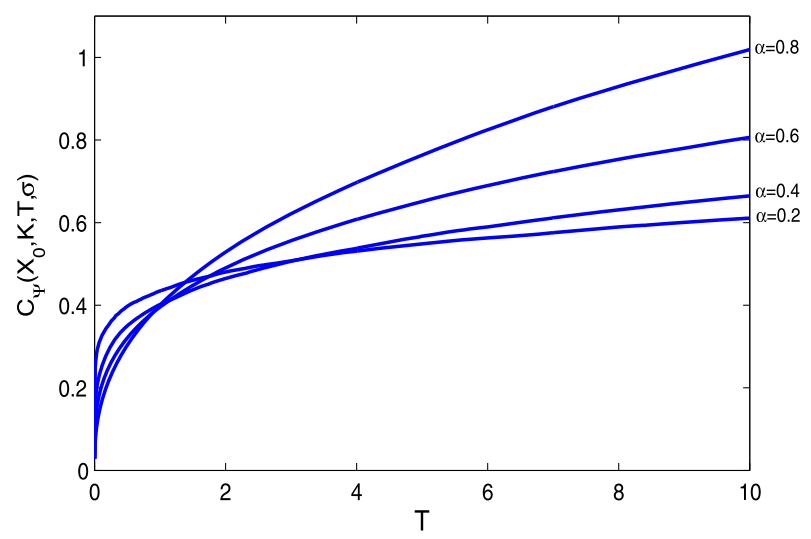

broad family of nonnegative id distributions. We have shown that the subdiffusive model is arbitrage-free and incomplete. Moreover, we have found the formulas for the fair prices of European options and mentioned, how to approximate them numerically. A more detailed considerations were introduced for two significant classes of waiting-time distributions: $\alpha$ stable (Fig. 2) and tempered $\alpha$-stable (Figs. 3-6).

Calibration of the subdiffusive model is very similar to the standard procedure used for diffusion processes. One only needs to remove the waiting times (constant periods) from the trajectory of the subdiffusive ABM (cf. Fig. 1). In this way one obtains the trajectory of the standard ABM, which can be analyzed in the usual way. Moreover, the sequence of removed waiting times forms a sample from the underlying id distribution. This sample can be used to verify statistically the distribution of waiting times, [29]. 
Fig. 5 Bachelier prices of European call option $C_{\Psi}\left(X_{0}, K, T, \sigma\right)$, according to the exercise date $T$ and strike price $K$. In the subdiffusive model the waiting times follow the tempered stable law $\left(\Psi(u)=(u+\lambda)^{\alpha}-\lambda^{\alpha}\right)$. Here, $\alpha, \lambda, X_{0}, \sigma$ are fixed and equal $0.9,0.1,100,1$, respectively
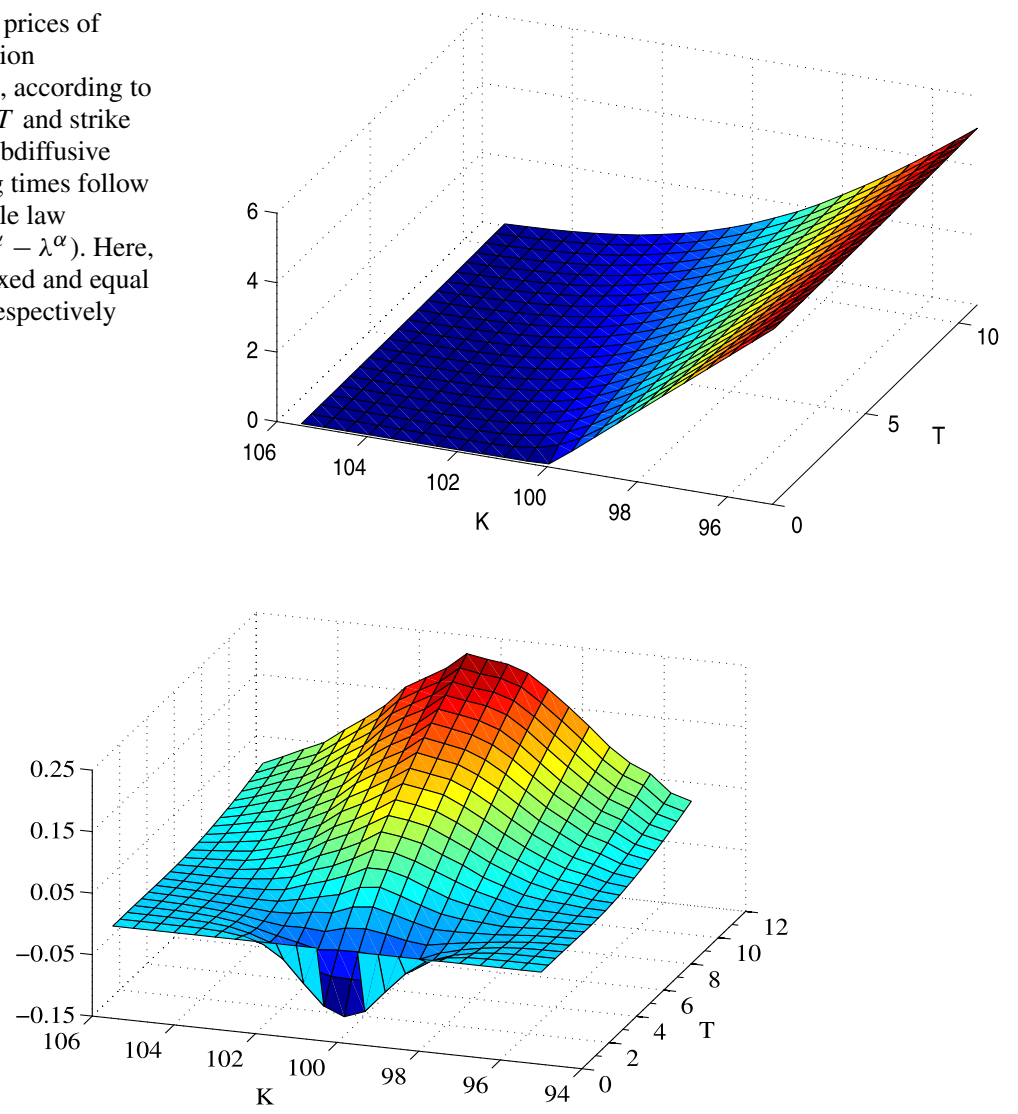

Fig. 6 The difference between the classical Bachelier price of the European call option and the subdiffusive one $C\left(X_{0}, K, T, \sigma\right)-C_{\Psi}\left(X_{0}, K, T, \sigma\right)$, according to the exercise date $T$ and strike price $K$. In the subdiffusive model the waiting times follow the tempered $\alpha$-stable law. Here, $X_{0}, \sigma, \alpha$ and $\lambda$ are fixed and equal $100,1,0.8$ and 0.1 respectively. For small $T$ the subdiffusive price is larger than the classical Bachelier price (the difference is negative), while for the larger $T$ the subdiffusive price is smaller than the Bachelier one

We believe that the introduced model will provide a more adequate description of the price processes especially in emerging markets, and that the derived pricing formulas will be applicable whenever the subdiffusive regime turns up.

We underline that the presented here methods and algorithms can be successfully employed to other diffusion processes in statistical mechanics. In this paper, for the sake of clarity and simplicity of the presentation, we concentrated only on ABM. Nonetheless, analogous methods can be applied to other diffusions time-changed by the large class of id inverse subordinators.

Acknowledgements The research of MM has been partially supported by the Polish Ministry of Science and Higher Education grant No. NN 201417639. The research of SO has been partially supported by the European Union within the European Social Fund.

Open Access This article is distributed under the terms of the Creative Commons Attribution Noncommercial License which permits any noncommercial use, distribution, and reproduction in any medium, provided the original author(s) and source are credited. 


\section{Appendix A}

DERIVATION OF THE FFPE (7). The process $X(\tau)$ is a standard ABM, thus its PDF $f(x, \tau)$ obeys the ordinary Fokker Planck equation

$$
\frac{\partial f(x, \tau)}{\partial \tau}=\left[-\mu \frac{\partial}{\partial x}+\frac{\sigma^{2}}{2} \frac{\partial^{2}}{\partial x^{2}}\right] f(x, \tau) .
$$

Let us introduce the notation

$$
L_{F P}=\left[-\mu \frac{\partial}{\partial x}+\frac{\sigma^{2}}{2} \frac{\partial^{2}}{\partial x^{2}}\right]
$$

The Laplace transform of (13) with respect to the variable $\tau$ has the form

$$
u \hat{f}(x, u)-f(x, 0)=L_{F P} \hat{f}(x, u) .
$$

Here, by $\hat{f}$ we denote the Laplace transform of $f$.

Next, 7 in the Laplace space $u$ yields

$$
u \hat{w}(x, u)-w(x, 0)=L_{F P} \frac{u}{\Psi(u)} \hat{w}(x, u) .
$$

We denote the PDFs of $T_{\Psi}(\tau)$ and $S_{\Psi}(t)$ by $h(t, \tau)$ and $g(\tau, t)$, respectively. By the property $\mathbb{P}\left(S_{\Psi}(t) \leq \tau\right)=\mathbb{P}\left(T_{\Psi}(\tau) \geq t\right)$, we obtain

$$
g(\tau, t)=-\frac{\partial}{\partial \tau} \int_{-\infty}^{t} h\left(t^{\prime}, \tau\right) d t^{\prime} .
$$

Consequently, by some standard calculations we obtain

$$
\hat{g}(\tau, u)=\frac{\Psi(u)}{u} e^{-\tau \Psi(u)} .
$$

Using the total probability formula and the independence of $X(\tau)$ and $S_{\Psi}(t)$, we obtain that the PDF $p(x, t)$ of $X\left(S_{\Psi}(t)\right)$ is given by

$$
p(x, t)=\int_{0}^{\infty} f(x, \tau) g(\tau, t) d \tau .
$$

Thus, its Laplace transform with respect to the variable $t$ is given by

$$
\hat{p}(x, u)=\frac{\Psi(u)}{u} \hat{f}(x, \Psi(u)) .
$$

Next, by the change of variables $u \rightarrow \Psi(u)$ in (14) we obtain

$$
\Psi(u) \hat{f}(x, \Psi(u))-f(x, 0)=L_{F P} \hat{f}(x, \Psi(u)) .
$$

Consequently, from (16) and the fact that $f(x, 0)=p(x, 0)$ we infer that $\hat{p}(x, u)$ satisfies the equation

$$
u \hat{p}(x, u)-p(x, 0)=L_{F P} \frac{u}{\Psi(u)} \hat{p}(x, u) .
$$

Comparing the above with (15) we have that $w(x, t)=p(x, t)$. This ends the proof. 


\section{Appendix B}

PROOF OF MARTINGALE PROPERTY FOR ABM $Y_{\Psi}(t)$. Let us introduce the following filtration

$$
\mathcal{G}_{t}=\mathcal{H}_{S_{\Psi}(t)}
$$

where

$$
\mathcal{H}_{\tau}=\bigcap_{u>\tau}\left\{\sigma(B(y): 0 \leq y \leq u) \vee \sigma\left(S_{\Psi}(y): y \geq 0\right)\right\}
$$

By analogous reasoning as Theorem 2.1 in [24], the processes $B\left(S_{\Psi}(t)\right)$ and $\exp \left\{-\gamma B\left(S_{\Psi}(t)\right)-\frac{\gamma^{2}}{2} S_{\Psi}(t)\right\}$ are martingales with respect to $\left(\mathcal{G}_{t}, \mathbb{P}\right)$. Here, $\gamma=\frac{\mu}{\sigma}$. We will show that

$$
K_{\Psi}(t)=B\left(S_{\Psi}(t)\right)+\gamma S_{\Psi}(t)
$$

is a $\left(\mathcal{G}_{t}, \mathbb{Q}\right)$-martingale, where $\mathbb{Q}$ is given by (8). Let us put

$$
H(t)=\left\langle\frac{d \mathbb{Q}}{d \mathbb{P}} \mid \mathcal{G}_{t}\right\rangle=\left\langle\exp \left\{-\gamma B\left(S_{\Psi}(T)\right)-\frac{\gamma^{2}}{2} S_{\Psi}(T)\right\} \mid \mathcal{G}_{t}\right\rangle .
$$

Here, $\left\langle. \mid \mathcal{G}_{t}\right\rangle$ denotes expectation conditioned by the history of the process up to time $t$, i.e. filtration $\mathcal{G}_{t}$. By the martingale property, we get that

$$
H(t)=\exp \left\{-\gamma B\left(S_{\Psi}(t)\right)-\frac{\gamma^{2}}{2} S_{\Psi}(t)\right\}
$$

or, equivalently

$$
d H(t)=-\gamma H(t) d B\left(S_{\Psi}(t)\right), \quad H(0)=1 .
$$

Moreover, the quadratic variation of $B\left(S_{\Psi}(t)\right)$ satisfies $\left[B\left(S_{\Psi}(t)\right), B\left(S_{\Psi}(t)\right)\right]=S_{\Psi}(t)$. Now, taking advantage of formula (19) and the Girsanov-Meyer theorem (see [31]), we get that the process

$$
\begin{aligned}
B & \left(S_{\Psi}(t)\right)-\int_{0}^{t} \frac{1}{H(s)} d\left\langle H(s), B\left(S_{\Psi}(s)\right)\right\rangle \\
& =B\left(S_{\Psi}(t)\right)+\gamma \int_{0}^{t} \frac{1}{H(s)} H(s) d\left\langle B\left(S_{\Psi}(s)\right), B\left(S_{\Psi}(s)\right)\right\rangle \\
& =B\left(S_{\Psi}(t)\right)+\gamma S_{\Psi}(t)=K_{\Psi}(t)
\end{aligned}
$$

is a local martingale with respect to $\mathbb{Q}$. Since $\left\langle\sup _{s \leq t}\left|K_{\Psi}(s)\right|\right\rangle_{\mathbb{Q}}<\infty, K_{\Psi}(t)$ is also a $\mathbb{Q}$ martingale. Consequently, $Y_{\Psi}(t)=\sigma K_{\Psi}(t)+X_{0}$ is a $\mathbb{Q}$-martingale.

\section{Appendix C}

FIRST PROOF OF INCOMPLETENESS OF THE MARKET MODEL. For every $\epsilon>0$, let us define the probability measure

$$
\mathbb{Q}_{\epsilon}(A)=C \int_{A} \exp \left\{-\gamma B\left(S_{\Psi}(T)\right)-\left(\epsilon+\frac{\gamma^{2}}{2}\right) S_{\Psi}(T)\right\} d \mathbb{P},
$$


where $C=\left(\left\langle\exp \left\{-\gamma B\left(S_{\Psi}(T)\right)-\left(\epsilon+\frac{\gamma^{2}}{2}\right) S_{\Psi}(T)\right\}\right\rangle\right)^{-1}$ is the normalizing constant, $\gamma=$ $\mu / \sigma$, and $A \in \mathcal{F}$.

For any $p>0$ and $n \in \mathbb{N}$, we have

$$
\begin{aligned}
\left\langle S_{\Psi}^{n}(t)\right\rangle & =\int_{0}^{\infty} x^{n-1} \mathbb{P}\left(S_{\Psi}(t)>x\right) d x=\int_{0}^{\infty} x^{n-1} \mathbb{P}\left(T_{\Psi}(x)<t\right) d x \\
& =\int_{0}^{\infty} x^{n-1} \mathbb{P}\left(e^{-p T_{\Psi}(x)}>e^{-p t}\right) d x \leq e^{p t} \int_{0}^{\infty} x^{n-1} e^{-x \Psi(p)}=\frac{e^{p t} \Gamma(n)}{\Psi^{n}(p)} .
\end{aligned}
$$

Thus, for $\lambda>0$ we get

$$
\left\langle e^{\lambda S_{\Psi}(t)}\right\rangle=\sum_{n=1}^{\infty} \frac{\lambda^{n}\left\langle S_{\Psi}^{n}(t)\right\rangle}{n !} \leq e^{p t} \sum_{n=1}^{\infty} \frac{\lambda^{n} \Gamma(n)}{\Psi^{n}(p) n !}=e^{p t} \sum_{n=1}^{\infty} \frac{\lambda^{n}}{\Psi^{n}(p) n}<\infty
$$

for large enough $p$ (recall that $\Psi(p) \rightarrow \infty$ as $p \rightarrow \infty$ ). This shows that the exponential moments of inverse subordinators are finite. Thus, the normalizing constant $C$ in the definition of $\mathbb{Q}_{\epsilon}$ is finite. Clearly, $\mathbb{Q}_{\epsilon}$ is equivalent to $\mathbb{P}$.

Let us put

$$
Y(t)=\exp \left\{-\gamma B(t)-\frac{\gamma^{2}}{2} t\right\}, \quad Z(t)=\sigma B(t)+\mu t
$$

We will show that $Y(t) Z(t)$ is an $\left(\mathcal{H}_{t}, \mathbb{P}\right)$-martingale, where $\left(\mathcal{H}_{t}\right)$ is defined in (18). Let $s<t$ and $A \in \mathcal{H}_{s}$. Let $\widehat{\mathbb{Q}}$ be the martingale measure corresponding to the classical Bachelier model $\left(\frac{d \widehat{\mathbb{Q}}}{d \mathbb{P}}=\exp \left(-\gamma B(T)-\frac{\gamma^{2}}{2} T\right)\right)$. We have

$$
\begin{aligned}
\left\langle Y(t) Z(t) \mathbf{1}_{A}\right\rangle & =\left\langle\mathbf{1}_{A} Z(t) Y(t) / Y(T)\right\rangle_{\widehat{\mathbb{Q}}} \\
& =\left\langle\mathbf{1}_{A} Z(t)\right\rangle_{\widehat{\mathbb{Q}}}=\left\langle\mathbf{1}_{A} Z(s)\right\rangle_{\widehat{\mathbb{Q}}}=\left\langle Y(s) Z(s) \mathbf{1}_{A}\right\rangle .
\end{aligned}
$$

Thus, $Y(t) Z(t)$ is an $\left(\mathcal{H}_{t}, \mathbb{P}\right)$-martingale.

Let us define $Z^{S_{\Psi}(T)}(t)=Z\left(t \wedge S_{\Psi}(T)\right)$. Then, the stopped process $Y\left(t \wedge S_{\Psi}(T)\right) Z^{S_{\Psi}(T)}(t)$ is also an $\left(\mathcal{H}_{t}, \mathbb{P}\right)$-martingale. Since the bounded random variable $e^{-\epsilon S_{\Psi}(T)}$ is $\mathcal{H}_{0}$-measurable, therefore

$$
\left(e^{-\epsilon S_{\Psi}(T)} Y\left(t \wedge S_{\Psi}(T)\right) Z^{S_{\Psi}(T)}(t)\right)_{t \geq 0}
$$

is an $\left(\mathcal{H}_{t}, \mathbb{P}\right)$-martingale as well. Additionally, for any $A \in \mathcal{H}_{t}$ we have

$$
\mathbb{Q}_{\epsilon}(A)=\left\langle\mathbf{1}_{A} e^{-\epsilon S_{\Psi}(T)} Y\left(t \wedge S_{\Psi}(T)\right)\right\rangle .
$$

Using the above result one can verify that $Z^{S_{\Psi}(T)}(t)$ is an $\left(\mathcal{H}_{t}, \mathbb{Q}_{\epsilon}\right)$-martingale. Moreover

$$
\begin{aligned}
\left\langle\sup _{t \geq 0} Z^{S_{\Psi}(T)}(t)\right\rangle_{\mathbb{Q}_{\epsilon}} & =\left\langle\sup _{t \leq S_{\Psi}(T)} Z(t)\right\rangle_{\mathbb{Q}_{\epsilon}} \\
& =\left\langle\exp \left\{-\gamma B\left(S_{\Psi}(T)\right)-\left(\epsilon+\frac{\gamma^{2}}{2}\right) S_{\Psi}(T)\right\} \sup _{t \leq S_{\Psi}(T)} Z(t)\right\rangle<\infty,
\end{aligned}
$$

which follows from Hölder inequality, Doob's maximal inequality and (21). 
Thus, $Z^{S_{\Psi}(T)}(t)$ is a uniformly integrable $\left(\mathcal{H}_{t}, \mathbb{Q}_{\epsilon}\right)$-martingale. It follows that there exists a random variable $X$ such that $Z^{S_{\Psi}(T)}(t)=\left\langle X \mid \mathcal{H}_{t}\right\rangle_{\mathbb{Q}_{\epsilon}}$. Finally, we get that $Z^{S_{\Psi}(T)}\left(S_{\Psi}(t)\right)=$ $\sigma B\left(S_{\Psi}(t)\right)+\mu S_{\Psi}(t)$ and also $Y_{\Psi}(t)=X_{0}+\sigma B\left(S_{\Psi}(t)\right)+\mu S_{\Psi}(t)$ are $\left(\mathcal{H}_{S_{\Psi}(t)}, \mathbb{Q}_{\epsilon}\right)$ martingales.

SECOND PROOF OF INCOMPLETENESS OF THE MARKET MODEL. We will show that not every random variable (contingent claim) $X \in \mathcal{G}_{T}$, such that $\left\langle X^{2}\right\rangle_{\mathbb{Q}}<\infty$, can be replicated by a self-financing strategy.

Let us assume that the contingent claim $X$ is a function of the stock price at time $T$, $X=h\left(Y_{\Psi}(T)\right)$. Moreover, let us assume that $h(x)$ is a continuous function $h: \mathbb{R} \rightarrow \mathbb{R}_{+}$such that $\left\langle h^{2}\left(Y_{\Psi}(T)\right)\right\rangle_{\mathbb{Q}}<\infty$ and $h^{\prime \prime}(x) \neq 0$ for some $x \in \mathbb{R}$. Now, let us assume (a contrario) that there exists a replicating, self-financing strategy for $X=h\left(Y_{\Psi}(T)\right)$. Consequently, the derivative payoff function $C_{t}=f\left(Y_{\Psi}(t), t\right)$ (where $f(y, t)$ is a smooth function) at time $t$ is given by

$$
f\left(Y_{\Psi}(t), t\right)=V_{t}(\phi)=a_{t} Y_{\Psi}(t)+b_{t} \beta(t)
$$

and

$$
C_{T}=f\left(Y_{\Psi}(T), T\right)=a_{T} Y_{\Psi}(T)+b_{T} \beta(T)=h\left(Y_{\Psi}(T)\right),
$$

where $\phi=\left(a_{t}, b_{t}\right)=\left(a\left(Y_{\Psi}(t), t\right), b\left(Y_{\Psi}(t), t\right)\right)$ is a trading strategy. Taking advantage of the self-financing condition we have

$$
d f\left(Y_{\Psi}(t), t\right)=d V_{t}(\phi)=a_{t} d Y_{\Psi}(t)+b_{t} d \beta(t) .
$$

From the stock price model and by the fact that $\beta \equiv 1$, we have

$$
d f\left(Y_{\Psi}(t), t\right)=a_{t} \mu d S_{\Psi}(t)+a_{t} \sigma d B\left(S_{\Psi}(t)\right)+0 d t .
$$

Now, by the Itô formula for semimartingales we get

$$
\begin{aligned}
d f\left(Y_{\Psi}(t), t\right)= & f_{t}\left(Y_{\Psi}(t), t\right) d t+\sigma f_{y}\left(Y_{\Psi}(t), t\right) d B\left(S_{\Psi}(t)\right) \\
& +\left(\mu f_{y}\left(Y_{\Psi}(t), t\right)+\frac{1}{2} \sigma^{2} f_{y y}\left(Y_{\Psi}(t), t\right)\right) d S_{\Psi}(t) .
\end{aligned}
$$

Now, comparing the coefficients from (24) and (25), we find that

$$
a_{t}=f_{y}\left(Y_{\Psi}(t), t\right), \quad f_{y y}\left(Y_{\Psi}(t), t\right)=0, \quad f_{t}\left(Y_{\Psi}(t), t\right)=0 .
$$

Consequently, replacing $Y_{\Psi}(t)$ by $x$ in (22), (23) and using formulas (26), we obtain the following system of equations:

$$
f(x, t)=x f_{x}(x, t)+b(x, t), \quad f(x, T)=h(x), \quad f_{x x}(x, t)=0 .
$$

From the third condition we find the general formula for $f(x, t)$ :

$$
f(x, t)=C_{1}(t) x+C_{2}(t),
$$

from the second condition, we get that at $t=T$ :

$$
C_{1}(T) x+C_{2}(T)=f(x, T)=h(x) .
$$


It means that $h^{\prime \prime}(x)=0$ for all $x \in \mathbb{R}$. This contradicts the assumption that $h^{\prime \prime}(x) \neq 0$ for some $x \in \mathbb{R}$. Therefore, not every contingent claim can be replicated, which implies that the subdiffusive Bachelier market is incomplete.

\section{References}

1. Bachelier, L.: Théorie de la spéculation. Ann. Éc. Norm. Super. 17, 21-86 (1900)

2. Baeumer, B., Meerschaert, M.M.: Tempered stable Lévy motion and transient super-diffusion. J. Comput. Appl. Math. 233, 2438-2448 (2010)

3. Bertoin, J.: Lévy Processes. Cambridge University Press, Cambridge (1996)

4. Black, F., Scholes, M.: The pricing of options and corporate liabilities. J. Polit. Econ. 81, 637-654 (1973)

5. Cartea, Á., del-Castillo-Negrete, D.: Fluid limit of the continuous-time random walk with general Lévy jump distribution functions. Phys. Rev. E 76, 041105 (2007)

6. Carr, P., Geman, H., Madan, D.B., Yor, M.: Self-decomposability and option pricing. Math. Finance 17, $31-51(2007)$

7. Cont, R., Tankov, P.: Financial Modeling with Jump Processes. Chapman \& Hall/CRC, London (2004)

8. Courtault, J.M., et al.: Louis Bachelier's on the centenary of Théorie de la Spéculation. Math. Finance 10, 341-353 (2000)

9. Deng, W.: Finite element method for the space and time fractional Fokker-Planck equation. SIAM J. Numer. Anal. 47, 204-226 (2008)

10. Dybiec, B.: Anomalous diffusion on finite intervals. J. Stat. Mech., P01011 (2010)

11. Dybiec, B.: Approaching stationarity: competition between long jumps and long waiting times. J. Stat. Mech., P03019 (2010)

12. Dybiec, B., Gudowska-Nowak, E.: Discriminating between normal and anomalous random walks. Phys. Rev. E 80, 061122 (2009)

13. Dybiec, B., Sokolov, I.M., Checkin, A.V.: Stationary states in single-well potentials under symmetric Lévy noises. J. Stat. Mech., P07008 (2010)

14. Einstein, A.: Über die von der molekularkinetischen Theorie der Wärme geforderte Bewegung von in ruhenden Flüssigkeiten suspendierten Teilchen. Ann. Phys. (Leipz.) 17, 549-560 (1905)

15. Galloway, M.L., Nolder, C.A.: Subordination, self-similarity, and option pricing. J. Appl. Math. Decis. Sci., 397028 (2008)

16. Itô, K.: On stochastic processes. Jpn. J. Math. 18, 261-301 (1942)

17. Janczura, J., Wyłomańska, A.: Subdynamics of financial data from fractional Fokker-Planck equation. Acta Phys. Pol. B 40(5), 1341-1351 (2009)

18. Janczura, J., Orzeł, S., Wyłomańska, A.: Subordinated Ornstein-Uhlenbeck process as a tool of financial data description. Preprint (2011)

19. Janicki, A., Weron, A.: A Simulation and Chaotic Behavior of $\alpha$-Stable Stochatic Processes. Dekker, New York (1994)

20. Kullback, S., Leibler, R.A.: On information and sufficiency Ann. Math. Stat. 22(1), 79-86 (1951)

21. Lévy, P.: Processus stochastiques at mouvement Brownian. Gauthier-Villars, Paris (1948)

22. Magdziarz, M.: Langevin picture of subdiffusion with infinitely divisible waiting times. J. Stat. Phys. 135, 763-772 (2009)

23. Magdziarz, M.: Black-Scholes formula in subdiffusive regime. J. Stat. Phys. 136, 553-564 (2009)

24. Magdziarz, M.: Path properties of subdiffusion-a martingale approach. Stoch. Models 26, 256-271 (2010)

25. Magdziarz, M., Weron, A., Weron, K.: Fractional Fokker-Planck dynamics: stochastic representation and computer simulation. Phys. Rev. E 75, 016708 (2007)

26. Merton, R.C.: Theory of rational option pricing. Bell J. Econ. Manag. Sci. 4, 141-183 (1973)

27. Metzler, R., Klafter, J.: The random walk's guide to anomalous diffusion: a fractional dynamics approach. Phys. Rep. 339, 1 (2000)

28. Musiela, M., Rutkowski, M.: Martingale Methods in Financial Modelling. Springer, Berlin (2005)

29. Orzeł, S., Weron, A.: Calibration of the subdiffusive Black-Scholes model. Acta Phys. Pol. B 41(5), 1051-1059 (2010)

30. Orzeł, S., Wyłomańska, A.: Calibration of the subdiffusive arithmetic Brownian motion with tempered stable waiting-times. J. Stat. Phys. 143(3), 447-454 (2011). doi:10.1007/s10955-011-0191-1

31. Protter, P.: Stochastic Integration and Differential Equations. A New Approach. Springer, Berlin/ Heidelberg (1990)

32. Rosinski, J.: Tempering stable processes. Stoch. Process. Appl. 117, 677-707 (2007) 
33. Rosinski, J.: Simulation of Lévy processes. In: Encyclopedia of Statistics in Quality and Reliability: Computationally Intensive Methods and Simulation. Wiley, New York (2008)

34. Samko, S.G., Kilbas, A.A., Maritchev, D.I.: Integrals and Derivatives of the Fractional Order and Some of Their Applications. Gordon \& Breach, Amsterdam (1993)

35. Samorodnitsky, G., Taqqu, M.S.: Stable Non-Gaussian Random Processes: Stochastic Models with Infinite Variance. Chapman \& Hall, New York (1994)

36. Samuelson, P.A.: Rational theory of warrant pricing. Ind. Manage. Rev. 6, 13-31 (1965)

37. Schachermayer, W., Teichmann, J.: How close are the option pricing formulas of Bachelier and BlackMerton-Scholes? Math. Finance 18, 155-170 (2008)

38. Smoluchowski, M.: Sur le chemin moyen parcouru par les molécules dún gaz et sur son rapport avec la théorie de la diffusion. Bull. Int. Acad. Sci. Cracovie, 202-213 (1906)

39. Stanislavsky, A.A.: Black-Scholes model under subordination. Physica A 318, 469-474 (2003)

40. Weron, R.: On the Chambers-Mallows-Stuck method for simulating skewed stable random variables. Stat. Probab. Lett. 28, 165-171 (1996)

41. Weron, A., Magdziarz, M.: Anomalous diffusion and semimartingales. Europhys. Lett. 86, 60010 (2009)

42. Wiener, N.: The average of an analytic functional and the Brownian movement. Proc. Natl. Acad. Sci. USA 7, 294-298 (1921) 